\title{
The impact of cognitive decline on stroke rehabilitation
}

\section{Fătu Ana-Maria ${ }^{1}$, Pâslaru Ana Maria ${ }^{1}$, Creangă-Zărnescu Valerica ${ }^{1}$, Mihaela Lungu ${ }^{1,2}$, Verenca Mădălina Codruţa ${ }^{1,3}$, Ciubară Anamaria ${ }^{1,4}$}

Corresponding author: Creangă-Zărnescu Valerica, E-mail: zarnescu vally@yahoo.com

2. Clinical Emergency Hospital "Sf. Apostol Andrei", Department Neurology, Galati, Romania 3. Clinical Emergency Hospital for Children "Sf.Ioan" , Department Rehabilitation, Galati, Romania 4. Psychiatry Hospital "Elisabeta Doamna", Galati, Romania

\begin{abstract}
Introduction. Stroke is a disease of great public importance, the second leading cause of death and the third leading cause of disability .The persistent and progressive decline of cognitive function in stroke survivors has a major impact on the patient's health and quality of life, with repercussions affecting the whole society. There is no cure for cognitive impairment or dementia, apart from the non-pharmacological treatment meaning the rehabilitation, which may be an important effective alternative.

Material and method. We performed a retrospective study for a period of 6 months. The patient population consisted of 60 individuals. Their primary diagnosis was stroke and the secondary was dementia. Patient assessment was done by clinical, imagistic and psychiatric examination. In addition, an analysis of randomized controlled data trials from the literature, on cognitive rehabilitation had been performed.

Results and discussions. The results show that $83,33 \%$ of patients were diagnosed with mixed dementia but $60 \%$ had this diagnosis before brain damage occured, with a Mini Mental State Examination under 20 points. The most common disorders of cognition were: fixation hypomnesia, temporal-spatial disorientation, impaired computing. Dementia patients have different rehabilitation needs and associate a lower functional result than those without cognitive impairments. For a rehabilitation benefit, patients should be able to recover their skills step by step and carry them over to the next session, which is difficult for people with severe memory problems. They have a low potential for progress and require a long time and many resources to advance with neurorehabilitation.

Conclusions. The brain injury, such as stroke, causes damage of motor and cognitive functions with repercussion on quality of life. The cognitive decline associated with stroke is an important aspect of neurorehabilitation. Nevertheless is slightly neglected to the detriment of physical disability as there is a lack of recovery potential in these patients.
\end{abstract}

Key words: dementia, stroke, cognitive, neurorehabilitation,

\section{Introduction}

Worldwide, cerebrovascular accidents are the second leading cause of death and the third leading cause of disability (1).

In 2017, the Stroke Alliance for Europe (SAFE) with the European Stroke Organisation (ESO), launched a comprehensive overview of stroke in Europe and they showed that between 2015 and 2035 the number of strokes is expected to rise by $34 \%$ due to an ageing population (2).

The life expectancy of patients with stroke has increased due to the fast application of an advanced treatment, but the aging population increases the incidence of cognitive decline. Also, the sudden death of brain cells due to lack of oxygen when the blood flow to the brain is lost by blockage or rupture of an artery, is a major cause of dementia (3). Thus,

the two entities are associated quite frequently, especially in the over-60 age group.

Current evidence suggests that $25-30 \%$ of stroke survivors develop immediate or delayed vascular cognitive disorders leading to dementia (4). Therewith $10 \%$ of patients have a diagnosis of dementia prior to first stroke, one third of patients develop dementia after recurrent stroke and many others may have undiagnosed pre-stroke cognitive impairment (5).

Patients with stroke who have cognitive impairments in addition to physical impairments have less recovery of physical function, more dependence in living after stroke (6), growth risk of mortality lower the quality of life. 


\section{Material and method}

We performed a six-month retrospective study in the Department of Neurology at the Emergency County Hospital "St. Apostol Andrei" Galati, in which we included 60 patients with main diagnosis of stroke, and secondary diagnosis of dementia. We have analyzed correlation between computer tomography images corresponding to brain damage and the severity of cognitive decline associated measured with Mini Mental State Examination (MMSE).

\section{Results and discussions}

During 6 months, a number (n) of 463 patients suffered a stroke and 60 patients had secondary diagnosis of vascular, mixed dementia or Alzheimer's disease. We included in our study only the patients who associated stroke and dementia.

We would like to draw the attention to the two terms: post stroke dementia (PSD) and vascular dementia (VD). Post-stroke dementia involves both neurodegenerative and vascular processes, that means a mixed dementia, while vascular dementia develops after a vascular event that can be highlighted on the neuroimaging, computer tomography $(\mathrm{CT})$ or magnetic resonance imaging (MRI). VD represents a concept which includes not only multiple cortical and/or subcortical infarcts, but also strategic single infarcts, non-infarction white matter lesions, hemorrhages, and hypoperfusion as possible causes of dementia (7).

In order to be able to make a proper classification, we must know the cognitive state before the injury. We found that $83,33 \%$ (50 patients) were diagnosed with mixed vascular dementia, 11,67\% (7 patients) with vascular dementia and 5\% (3 patients) with Alzheimer's disease.

Stroke can facilitate the onset of vascular dementia as well as aggravate pre-existing cognitive decline that manifest immediately, within three months of the event. In contrast, there are cases that develop dementia delayed or only after recurrent stroke. This delay can be seen as a therapeutic time window allowing interventions to be applied to preserve cognition following stroke (8).

During the study, $53,33 \%$ of patients $(n=32)$ had a diagnosis of dementia before the onset of brain injury, and $41,66 \%$ of patients $(n=25)$ had recurrent cerebral infarctions. It is a fairly high percentage of recurrent vascular accidents, which argues that neurodegeneration predisposes to new brain damage. Recurrent stroke can be prevented by drug intervention, but post-stroke dementia can't be slowed down with any medication. It can be tried with a secondary prevention that involves modifiable risk factors like: healthy diet, regular exercise, reduce the chronic stress, social interaction. Pre-existing dementia is associated with higher levels of disability and risk of death (9). They have a bigger probability to be institutionalized after stroke comparing to patients without pre-existing dementia (9).

A study comparing short-term outcomes for 919 stroke patients found that whilst the $11.5 \%$ of patients with pre-existing dementia had a higher level of disability at baseline and discharge than patients without (10). They made similar daily gains in motor function as measured using the Functional Independence Measure (FIM), a validated tool within stroke rehabilitation (10).

Skills such as: abstract thinking, judgment, shortterm verbal memory, understanding and guidance are important to predict the functional status of the stroke survivor.

Regarding the stroke type, patients with ischemic strokes usually have higher survival rates than those with hemorrhagic strokes, which explains why ischemic strokes lead to psychiatric morbidity more frequently than do hemorrhagic strokes (11).

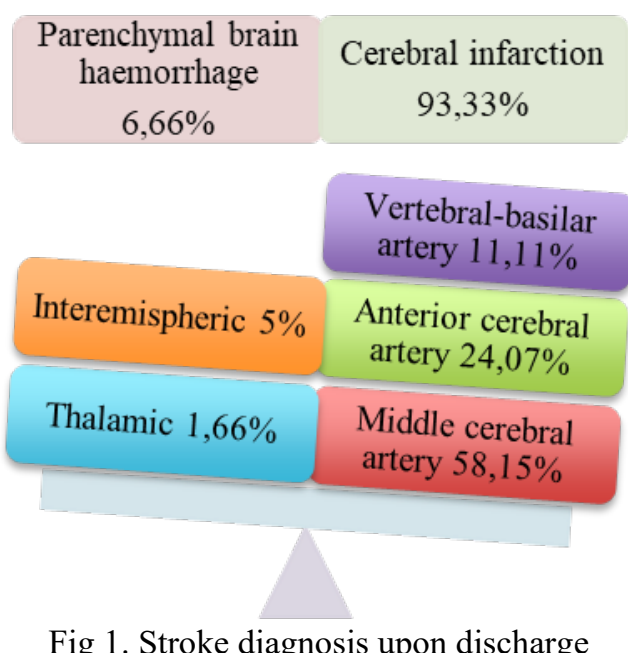

In our study parenchymal brain hemorrhage had $6,66 \% \quad(n=4)$ of patients, 3 patients with interhemispheric and 1 patient with thalamic hemorrhage. Cerebral infarction had 93,33\% patients $(\mathrm{n}=56)$, anterior cerebral artery was involved in $24,07 \%$ cases $(n=13)$ and the middle cerebral artery in $58,15 \%$ cases $(n=37)$, with $56,75 \%(n=21)$ on the right side and $43,25 \%(n=16)$ on the left side.

In a right hemisphere middle cerebral infarct, visualspatial perceptual disorders include left-sided neglect, figure ground disorientation, constructional 
apraxia and astereognosis (12).

Thus patients with unilateral neglect syndrome, ignore the left side of the body, are disorientated, do not respond to sensory stimuli related to the affected part, they forget to use their hand when they dressed, when they eat. At the same time, these patients may associate anosognosia. They can get injured because they are not aware of the existing deficit on the affected side. Hier et al. found that after right hemispheric lesions, recovery from unilateral neglect and anosognosia was the most rapid (13).

Patients who had lesion in the middle cerebral artery on the left hemisphere, presented expressive, motor or mixed aphasia in $33,33 \%$ of cases $(n=20)$ and $15 \%$ of cases $(n=9)$ presented apraxia. The disorder of the voluntary movement and the problems with understanding of language constitute a series of obstacles in carrying out the recovery plan.

Vascular brain injury and the resulting cellular damage (oxidative stress, swelling) appear to be the causes of the altered brain ageing process, leading to increased risk for stroke, cognitive decline, dementia, depression, and other neurological problems, such as gait disorders (14).

The prevalence of post-stroke cognitive decline has a broad range, from $20 \%$ to $80 \%$ (11). This variability depends on the diagnostic tools and the criteria employed, on the extent of the vascular and neurodegenerative pathologies before the stroke, on the cognitive status, on the stroke extension (volume) and topography (strategic areas such as frontal cortex, hippocampus or white matter) (15).

Dementia imaging provides important information about the anatomical substrate of the condition and can assess the degree of cognitive decline.

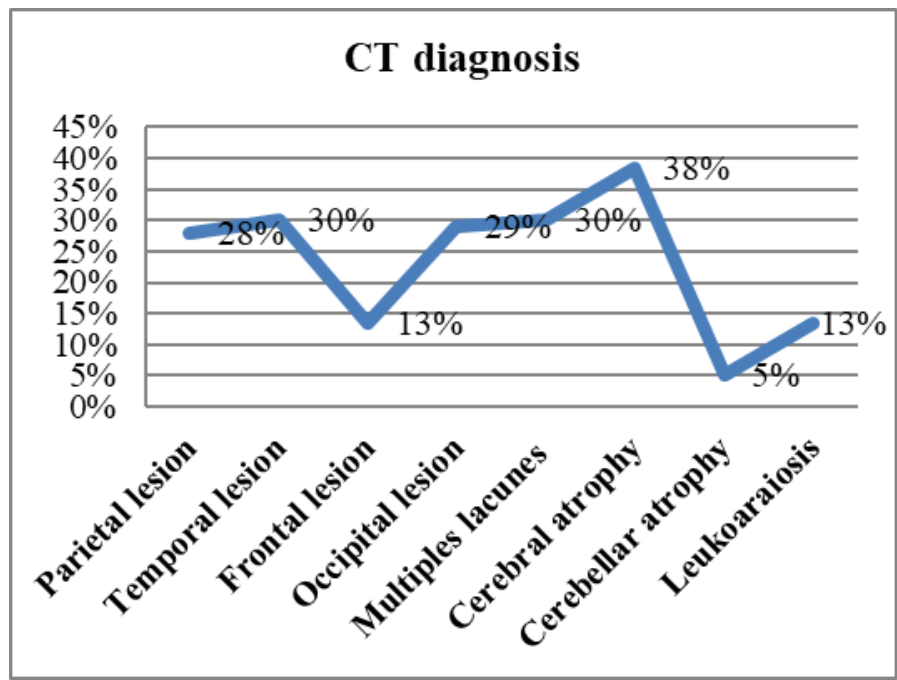

Fig 2. Different cerebrovascular pathologies associated with dementia- CT diagnosis
In figure 2 it is noticed that $30 \%$ of patients $(n=17)$ had temporal lesion and in 6 cases was affected the dominant medial temporal lob, that means this pacients developed immediate cognitive syndrome.

Strategic infarct dementia is attributed to locations in the angular gyrus, the medial frontal lobe, and the inferomedial portion of the temporal lobe (16).

Frontal lesion was observed in $13,33 \%$ of cases $(n=8)$ with repercussion on the executive function manifested by reducing the abilities: to control their impulses, to organize their thoughts, to change their priorities, to evaluate their progress. The functions normally associated with the frontal lobe are intellect, emotions, behaviour, language, personality, control centers for higher autonomic functions, abstract thinking and motor movement (7). The patients became emotional, impatient, rigid, with difficulties in concentrating and following the instructions given by the therapists. All of this can influence but also can predict the potential of the functional recovery.

A study of 663 occupational therapists working in stroke services in Canada found that when treating post-stroke cognitive impairment most therapists did not address executive function, which is often affected by vascular dementia (17).

Even more, there are exclusions to rehabilitation services in every international clinical guidelines. For example, in Spanish guidelines, rehabilitation is not recommended for patients with severe stroke and poor recovery prognosis (18). In Canada, patients must demonstrate the potential ability to return to pre-stroke levels of function or to increase poststroke functional level (19). Given these issues, the patient with stroke and severe cognitive decline can't be a candidate for rehabilitation, but we believe that any minor gain that can improve their functional independence is a success in terms of growth the quality of life. Rehabilitation potential is difficult to predict due to the fact that some patients demonstrate their potential later than others (20).

Patients included in our study, with severe cognitive impairment, associated multiple CT images: $30 \%$ $(\mathrm{n}=18)$ silent lacunar infarctions, 38,33\% $(\mathrm{n}=23)$ cerebral atrophy predominantly in the hippocampus, $13,33 \%(n=8)$ lesions of the periventricular white matter.

The presence of silent multiple lacunar infarctions in patients with first-ever lacunar stroke is an independent predictor of poor performance on executive functions and short delayed verbal 
memory tests (7). According to study of GrauOlivares and Arboix ischemic cerebral small-vessel disease (SVD) should be regarded as a severe condition prodrome of subcortical VD, rather than a relatively benign disorder (21).

Bilateral hippocampal or thalamic infarctions and unilateral thalamic infarctions are other examples of strategically localized infarctions that are reported to cause dementia (22). In the hippocampal lesions the anterograde memory predominated. The patients were not able to accumulate new information, to store the recent experiences, to remember the natural course of the sequence of the tasks, all of these are necessary for the rehabilitation process. When thalamus lesions were also associated, patients suffered from retrograde amnesia. They can't remember their name, the familiar faces, the home.

A systematic review of the prevalence of memory impairment after stroke estimated that between $23 \%$ and $55 \%$ have memory impairment three months after stroke and between $11 \%$ and $31 \%$ a year after stroke (23). Cumming et al. notes that at one year post stroke, a majority of patients still had attention deficits, while deficits in language and memory were more likely to have resolved (24).

The most commonly used assessments for cognitive impairment or dementia after stroke are MMSE and Montreal Cognitive Assessment (MoCA). The MMSE evaluates especially dementia and we used it in our study: $40 \%$ of patients $(n=24)$ had an MMSE score below 9 points, indicating a severe impairment of cognitive functions. Stroke patients with low scores are at high risk of dementia over time, even in the absence of recurrent stroke and should therefore be closely monitored for further cognitive decline.

Even minor stroke affects daily functioning, executive functions, and cognition, consequently affecting quality of life and return to work (7).

In the presented study, following the psychological examination the most common disorders of cognition were: fixation and evocation hypomnesia, temporal-spatial disorientation, disorders of mental calculation. It is difficult to work with these patients who are not aware of time or orientation in space. They can become anxious if they do not understand where they are or their purpose in that place.

In addition, cognitively impaired patients may not be able to benefit fully from rehabilitation because of their impairment. Research suggests that this may occur in patients with a sensory deficit (contralateral visual-neglect), attentional deficits or impaired comprehension and learning (6).

\section{Conclusion}

Brain injury through the infarction or cerebral hemorrhage, produces deficits consequences in motor and cognitive functions with repercussions on the rehabilitation plan. These patients have a low potential for progress, a reduced functional outcome implying different recovery needs and an individualized program, properly adapted to his deficits. They should be able to recover their skills step by step and carry them over to the next session, which is difficult for people with severe dementia.

Patients with severe cognitive impairment, objectively identified by a 9-point MMSE score, have associated multiple lacunar infarction, cerebral atrophy, periventricular white substrate lesions, and infarcts located strategically in the temporal or frontal lobe. It means that the severe dementia associates neurodegenerative process and vascular lesion.

The executive function, frequently affected in vascular dementia, recovered much harder than the motor one and has more psychosocial consequences causing permanent disability.

The cognitive decline associated with stroke is an important aspect of neurorehabilitation slightly neglected to the detriment of physical disability.

We need researches on the therapeutic plan of recovery of patients with stroke and dementia in order to increase their functional outcome and their level of independence.

There are no specific guidelines or recommendations regarding rehabilitation for these patients.

\section{Author contributions.}

The authors contributed equally to the work.

\section{Declaration of conflict of interests}

The authors declare that there is no conflict of interest regarding the publication of this article.

\section{Informed consent}

Informed consent was obtained from all patients included in this study.

\section{References}

1. Walter J, Oyere O, Mayowa O, Sonal S. Stroke: a global response is needed. Bulletin of the World Health Organization 2016; 94:634-634A. 
2. Europenean Stroke Organisation and Stroke Alliance of Europe. Stroke action plan for Europe 2018-2030. Brussels 2018. Available from https://www.safestroke.eu/wpcontent/uploads/2019/05/SAFE-SAPE-ebookcorrect-version_compressed-FINAL-FINAL.pdf

3. Owolabi MO, Akarolo-Anthony S, Akinyemi R, Arnett D, Gebregziabher M, Jenkins C, et al. The burden of stroke in Africa: a glance at the present and a glimpse into the future. Cardiovasc J Afr. 2015 Mar-Apr;26(2) Suppl 1:S27-38.

4. Kalaria RN, Akinyemi R, and Ihara M. (2016) Stroke injury, cognitive impairment and vascular dementia. Biochim Biophys Acta. 1862(5), 915925

5. Pendlebury ST \& Rothwell, P. M. (2009). Prevalence, incidence, and factors associated with pre-stroke and post-stroke dementia: a systematic review and meta-analysis. The Lancet Neurology, 8(11), 1006-1018.

6. Zinn S, Dudly TK, Bosworth HB, Hoenig HM, Duncan PW, Horner RD. The effect of poststroke cognitive impairment on rehabilitation process and functional outcome. Arch Phys Med Rehabil 2004;85:1084-90.

7. Mijajlović MD, Pavlović A, et all. Post-stroke dementia - a comprehensive review. BMC Med. 2017; 15: 11.

8. M. Brainin J, Tuomilehto WD et all. Post-stroke cognitive decline: an update and perspectives for clinical research. Europeanean Journal of Neurology. Volume 22, 2015, Pages 229-e16

9. Tatemichi TK, Paik M, Bagiella E, Desmond D W, Pirro M. \& Hanzawa LK. Dementia after stroke is a predictor of long-term survival. Stroke, 1994, 25(10), 1915-9

10. Mizrahi, E.-H., Arad, M. \& Adunsky, A. Prestroke dementia does not affect the post-acute care functional outcome of old patients with ischemic stroke. Geriatrics \& Gerontology International, 2016, 16(8), 928-933.

11. Augusto Vicario, Gustavo H Cerezo, At The Heart Of Brain Disorders Preventing Cognitive Decline And Dementia , Ecr, Volume 10, 2015, 62-68

12. Robert Teasell MD, Norhayati Hussein MBBS MRehabMed, Ricardo Viana MD, Sarah Donaldson BHSc, Mona Madady MSc . Clinical Consequences of Stroke . Stroke Rehabilitation Clinician Handbook 2016, 1-27

13. Hier DB, Mondlock J, Caplan LR. Behavioral abnormalities after right hemisphere stroke.
Neurology. 1983; 33(3):337-344 (a).Hier DB, Mondlock J, Caplan LR. Recovery of behavioural abnormalities after right hemisphere stroke. Neurology 1983; 33:345-350 (b).

14. Sun JH, Tan L, Yu JT. Post-stroke cognitive impairment: epidemiology, mechanism and management. Ann Transl Med. 2014;2:80-96

15. Onyike CU. Cerebrovascular disease and dementia. Int. Rev. Psychiatry. 2006;18:423-431

16. Goetter, W. Nursing diagnoses and interventions with the acute stroke patient.Nursing Clinics of North America (1986) 21(2), 309 \pm 19

17. Korner-Bitensky N, Barrett-Bernstein S, Bibas G \& Poulin V. (2011). National survey of Canadian occupational therapists' assessment and treatment of cognitive impairment post-stroke. Australian Occupational Therapy Journal, 58(4), 241-250.

18. Lynch EA, Cadilhac DA, Luker JA \& Hillier SL. (2017a). Inequities in access to inpatient rehabilitation after stroke: an international scoping review. Topics in Stroke Rehabilitation, 1-8.

19. Hebert D, Lindsay MP, Amanda M, Adam K, Peter GR, Stephen B et all (2016). Canadian stroke best practice recommendations: Stroke rehabilitation practice guidelines, update 2015 . International Journal of Stroke, 11(4), 459-484.

20. Enderby P, Pandyan A, Bowen A, Hearnden D, Ashburn A, Conroy P, Logan P, Thompson C \& Winter J. (2016). Accessing rehabilitation after stroke - a guessing game? Disability and Rehabilitation, 1-5.

21. Grau-Olivares M, Arboix A. Mild cognitive impairment in stroke patients with ischemic cerebral small-vessel disease: a forerunner of vascular dementia? Expert Rev Neurother. 2009;9(8):1201-1217. doi: 10.1586/ern.09.73

22. Raj N. Kalaria, Rufus Akinyemi, and Masafumi Ihara, Stroke injury, cognitive impairment and vascular dementia, Biochim Biophys Acta. 2016 May; 1862(5): 915-925

23. Snaphaan L, de Leeuw FE. Poststroke memory function in nondemented patients: a systematic review on frequency and neuroimaging correlates. Stroke. 2007 Jan;38(1):198-203.

24. Cumming TB, Marshall RS, Lazar RM. Stroke, cognitive deficits, and rehabilitation: still an incomplete picture. Int J Stroke 2013; 8(1):3845. 